\title{
Superamphiphobicity and electroactivity enabled dual physical/chemical protections in novel anticorrosive nanocomposite coatings
}

\author{
Ruixia Yuan ${ }^{\mathrm{a}, \mathrm{b}}$, Shiqi $\mathrm{Wu}^{\mathrm{a}}$, Baohui Wang ${ }^{\mathrm{a}}$, Zhanjian Liu ${ }^{\mathrm{a}}$, Liwen $\mathrm{Mu}^{\mathrm{b}}$, Tuo Ji ${ }^{\mathrm{b}}$, Long \\ Chen $^{\mathrm{b}}$, Bowen Liu ${ }^{\mathrm{a}}$, Huaiyuan Wang ${ }^{\mathrm{a} *}$, Jiahua $\mathrm{Zhu} \mathrm{u}^{\mathrm{a}, \mathrm{b} *}$ \\ ${ }^{a}$ Provincial Key Laboratory of Oil \& Gas Chemical Technology, College of Chemistry and \\ Chemical Engineering, Northeast Petroleum University, Daqing 163318, China \\ ${ }^{\mathrm{b}}$ Intelligent Composites Laboratory, Department of Chemical and Biomolecular Engineering, The \\ University of Akron, Akron, OH 44325, USA \\ Email: wanghyjiji@163.com (H. Wang); jzhu1 @uakron.edu (J. Zhu)
}

\begin{abstract}
Superamphiphobic and electroactive composite coating on aluminum substrate was successfully fabricated by integrating epoxy, polyaniline (PANI), fluorinated ethylene propylene, carbon nanotubes and $\mathrm{SiO}_{2}$ nanoparticles into a two-layer coating configuration with nano/micro hierarchical surface structure. The composite coating demonstrates strong adhesion, excellent wear resistance and durable anti-wettability. The electrochemical tests demonstrated significantly enhanced anticorrosion performance by surface barrier effect of the superamphiphobic coating and redox catalytic capability of embedded electroactive PANI. The design principle of the multi-functional coatings will definitely benefit the advancement of durable anti-corrosive coatings and the achieved superior properties will promise a wider engineering applications in petroleum related industries.
\end{abstract}

Keywords: polymer nanocomposites; superamphiphobic property; anti-corrosion coating 


\section{Introduction}

Despite the excellent physicochemical properties of aluminium (Al) and its alloys, such as high electrical conductivity, good processability, low density and ductility, their applications in aggressive aqueous conditions are greatly restricted due to potential corrosion [1,2]. In particular, chloride ions can easily breakdown the passivation film on aluminium surface and lead to initiation of pitting corrosion [3]. The traditional organic insulation coatings, such as epoxy, polyurethane, polyester, etc., are thermosetting resins and lack of long-term abrasion resistance. The corrosive chloride ions can attack the scratch or holes in the coating, then induce and accelerate under-coating localized corrosion due to small anode-large cathode galvanic corrosion reaction [4-6].

Conductive polymer polyaniline (PANI) has gained considerable attention recently because of their superior anticorrosion properties, unique redox tunability, environmental stability and ease of synthesis [7-10]. Besides physical barrier effect, PANI coating can also chemically protect metal substrate from corrosion by anodic shift in corrosion potential and catalytic activity in the formation of oxide passivation layer between metal and coating [11-13]. The chemical role of PANI is mainly due to its redox state and the highly delocalized electrons capable of transferring charges in the $\pi$-conjugated PANI chains $[14,15]$. Moreover, nano-structured PANI with unique quantum size effect and surface effect has demonstrated enhanced corrosion protection performance [4].

Unfortunately, the high porosity and intrinsic hydrophilicity of PANI coating results in fast penetration of small electrolyte ions such as chloride through coating layer and cause corrosion at the metal-coating interfaces $[14,16]$. These problems can be potentially alleviated by creating superhydrophobic surface [17]. Inspired by natural lotus leaves, the biomimetic superhydrophobic surface with water contact angle (WCA) greater than $150^{\circ}$ possesses extremely high water repellency, and thus suppresses the metal surface wetting by water [18-20]. Therefore, the propagation of corrosion can be effectively inhibited by blocking the diffusion of corrosive chloride 
ions and preventing the cathodic reaction $\left(2 \mathrm{H}_{2} \mathrm{O}+\mathrm{O}_{2}+4 \mathrm{e}^{-} \rightarrow 4 \mathrm{OH}^{-}\right)$beneath the coating [21].

It is expected that the superhydrophobic/electroactive PANI coating could provide enhanced anticorrosion performance with dual physical and chemical protections. Peng et al. firstly developed nanocasting technique using a natural leaf and polydimethylsiloxane (PDMS) as template to prepare superhydrophobic PANI surface which achieved better corrosion protection as compared to the flat PANI [22]. Kumar and Gasem fabricated hydrophobic PANI/functional carbon nanotubes (f-CNTs) composite coatings which showed a remarkable improvement in corrosion resistance of mild steel in $3.5 \mathrm{wt} \% \mathrm{NaCl}$ solution [16]. Nevertheless, the practical application of these bare PANI coatings is limited to their low mechanical strength, weak adhesion and poor wear-resistance [4]. Especially, coating surface will be unavoidably in contact with small particles, leading to abrasion and destruction of the hierarchical roughness features and therefore reduced anti-wettability [23-25]. Therefore, robust composite coating with superhydrophobic film-forming materials and conductive PANI nanofiller is proposed in this work to maintain the coating anticorrosion property.

Furthermore, the superhydrophobic coating can be still wetted by organic liquids such as surfactant-based solutions, alcohols or oily contaminants, especially in practical petroleum industry $[25,26]$. It is essentially important to fabricate coatings with both superhydrophobic and superoleophobic (superamphiphobic) properties to improve their corrosion resistance in harsh conditions [27,28]. To the best of our knowledge, the superamphiphobic/electroactive composite coating with embedded PANI has not been reported so far. Therefore, a facile, cost-effective and reproducible method is in urgent demand to develop a superamphiphobic/electroactive coating layer on aluminium with high stability and durability in corrosive medium from both theoretical and practical point of view.

Techniques for fabricating superamphiphobic surfaces include designing nano/micro hierarchical roughness on hydrophobic surfaces and modifying rough surfaces with low surface energy materials [29,30]. In this work, we successfully 
fabricated superamphiphobic/electroactive coatings on aluminium substrate by using simple air spraying and electrostatic spraying technologies. These spraying techniques are suitable to scale up for fabricating large and uniform areas. Conductive PANI was synthesized via chemical polymerization approach. Low-cost epoxy resin (EP) was employed with the benefits of its outstanding corrosion-resistance and thermal stability. Fluorinated ethylene propylene (FEP) was used because of its high chemical inertness, low surface energy $\left(17.8-18.8 \mathrm{~mJ} / \mathrm{m}^{2}\right)$ and unique self-lubrication property. The mechanical property of the coating was improved by the excellent cross-linking effect of epoxy resin reinforced by nanofillers, including CNTs, nano-sized $\mathrm{SiO}_{2}$, FEP and PANI. The durable non-wettability, wear resistance, adhesion ability and anti-corrosion behaviour of the as-prepared EP/PANI/FEP/CNTs/SiO 2 composite coatings in corrosive $\mathrm{NaCl}$ solution were investigated and the anticorrosion mechanisms were discussed. This research will create a new path to fabricate multi-functional nanocomposite coatings aiming for large-scale industrial applications.

\section{Experimental}

\subsection{Materials}

Commercial EP powder (E-42) was supplied by Qingdao Meiheng Co. Ltd (China). FEP and Multi-walled CNTs were purchased from DuPont (USA) and Beijing Boyu New Material Technology Co. Ltd (China), respectively. Aniline, hydrochloric acid $(\mathrm{HCl})$, sodium chloride, sodium hydroxide $(\mathrm{NaOH})$, ammonium persulfate (APS), ethanol and $\mathrm{SiO}_{2}$ particles with average diameter of $40 \mathrm{~nm}$ were obtained from Aladdin Reagent Co. Ltd (China).

\subsection{Preparation of PANI}

Synthesis of doped PANI: $0.233 \mathrm{~g}$ aniline was firstly added into $50 \mathrm{~mL}$ of $1.0 \mathrm{M}$ $\mathrm{HCl}$ with magnetic stirring to form a transparent aqueous solution. $0.571 \mathrm{~g}$ APS dissolved in $50 \mathrm{~mL}$ of $1.0 \mathrm{M} \mathrm{HCl}$ was then rapidly mixed with the above aniline solution. After stirring for $10 \mathrm{~min}$, the polymerization reaction was carried out at $0 \pm 1$ 
${ }^{\circ} \mathrm{C}$ for $24 \mathrm{~h}$ without any disturbance. With the addition of APS, gradual colour change from yellow, blue to dark green was observed. The resultant PANI was washed with distilled water and ethanol to remove aniline monomers and oligomers. Finally, the precipitate was dried at $60{ }^{\circ} \mathrm{C}$ in vacuum for $24 \mathrm{~h}$.

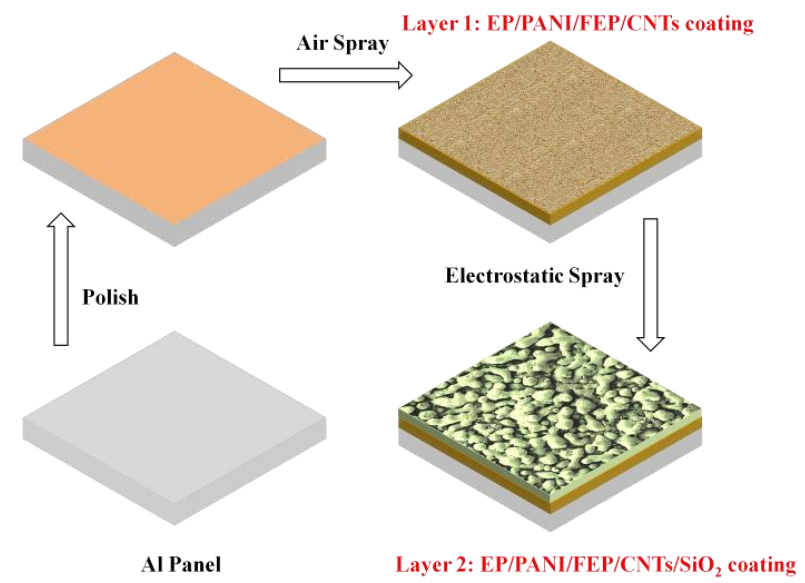

Fig. 1. Schematic illustration of the fabrication process for EP/PANI/FEP/CNTs/SiO 2 composite coating with dual nano/micro-scaled roughness.

\subsection{Preparation of the composite coatings}

The schematic diagram for the fabrication process of the nano/micro-structured composite coating is illustrated in Fig. 1 . The aluminum plate (1100 grade, $80 \mathrm{~mm} \times$ $80 \mathrm{~mm} \times 1 \mathrm{~mm}$ ) was first polished with 600 mesh sand papers in one direction, and then ultrasonically washed in anhydrous ethanol for $15 \mathrm{~min} .2 .0 \mathrm{~g}$ EP powders were dissolved in $15 \mathrm{~mL}$ ethyl acetate to form a well-dispersed solution by magnetic stirring at ambient temperature. Then, $0.05 \mathrm{~g}$ FEP powders, $0.05 \mathrm{~g}$ CNTs and certain amount of PANI were added into the EP solution and dispersed with ultrasonication for $30 \mathrm{~min}$. The solution was sprayed on the polished Al plate as the first layer using an air spray gun under a pressure of $6 \mathrm{bar}$, and then cured at $150{ }^{\circ} \mathrm{C}$ for $1 \mathrm{~h}$. The hydrophobic surface with WCA of $128^{\circ}$ was obtained (Fig. S1). After optimizing the experimental conditions (Fig. S2), $2.0 \mathrm{~g}$ EP powders, $0.2 \mathrm{~g}$ FEP, $0.05 \mathrm{~g}$ CNTs, $0.05 \mathrm{~g}$ $\mathrm{SiO}_{2}$, and the same amount of PANI material as the first layer were mixed adequately in a mix blender to obtain the uniform hydrophobic coating precursors. The mixture was sprayed with an electrostatic system onto the first layer. Finally, the composite 
coatings with internal nano/micro-structures were obtained after calcinated at $180{ }^{\circ} \mathrm{C}$ for $2 \mathrm{~h}$.

\subsection{Characterization}

The contact angles (CAs) and sliding angles (SAs) of the composite coatings were measured by Contact Angle Meter (JGW-360A, Chengdeshi Shipeng Detection Equipment Co. Ltd) with $5 \mu \mathrm{L}$ distilled water or glycerol. Average values were taken from 5 measurements at different positions of the coating surface. The coating surface morphology was observed by using a Zeiss SIGMA field emission scanning electron microscope (SEM). The surface roughness of the coatings was measured by a confocal microscope (OLYMPUS OLS-3000). The variation of WCAs and coating morphology were also investigated after immersing into strongly acidic $(\mathrm{pH}=1)$ and alkaline $(\mathrm{pH}=14)$ solutions for 90 days. The chemical composition and crystalline structure of the prepared coatings was characterized by Tensor27 infrared spectrometer (FT-IR), X-ray diffraction (XRD) (D/max 2200) and X-ray energy dispersive spectrometry (EDS) using an Oxford Instruments X-Max $80 \mathrm{~mm} 2$ silicon drift detector. The elemental composition of PANI was characterized by X-ray photoelectron spectroscopy (XPS). XPS was carried out on a PHI VersaProbe II Scanning XPS Microprobe using Al Ka line excitation source.

The adhesive strength of the composite coating was qualitatively determined by cross-cut method according to standard GB/T 9286. The wear-resistant property of the coating was estimated by rubbing with 1000 mesh sand papers, details refer to our previous report [29]. A pressure of 0.5 $\mathrm{MPa}$ was applied onto the sand paper which was moved in one direction on the coating.

\subsection{Electrochemical corrosion studies}

The electrochemical corrosion test was performed in $3.5 \mathrm{wt} \% \mathrm{NaCl}$ aqueous solution at room temperature using an electrochemical workstation (METEK VersaSTAR 4). Uncoated and coated Al plates with exposed surface area of $1 \times 1 \mathrm{~cm}$ were used as the working electrode, Pt wire as counter electrode and saturated 
calomel electrode (SCE) as reference electrode. All measurements were repeated at least three times to ensure the reproducibility and statistical significance. Open circuit potential (OCP) at the equilibrium state of the system was recorded as the corrosion potential ( $E_{\text {corr }}$ in $\mathrm{mV}$ versus SCE). The Tafel plots were obtained by scanning the potential from -250 to $250 \mathrm{mV}$ above the $E_{\text {corr }}$ at a scan rate of $50 \mathrm{mV} / \mathrm{min}$. The corrosion current $\left(I_{\text {corr }}\right)$ was determined through superimposing a straight line along the linear portion of the cathodic and anodic curves and extrapolating them through $E_{\text {corr }}[22]$. Electrochemical impedance spectroscopic (EIS) measurements were carried out in the frequency range from $100 \mathrm{kHz}$ to $10 \mathrm{mHz}$, with a sinusoidal signal perturbation of $10 \mathrm{mV}$. The experimental EIS spectra were interpreted based on equivalent electrical analogues using the ZSimpwin software program to obtain the fitting parameters. The corrosion rate $(\mathrm{CR})$ was calculated using $I_{\text {corr }}$ from the following equation [31].

$$
\mathrm{CR}=3270 \times \frac{I_{\text {corr }} \cdot \mathrm{M}}{\mathrm{V} \cdot \mathrm{d}}
$$

where 3270=0.01×[1 year (in s)/96 497.8] and 96 497.8=1 Faraday in Coulombs. In addition, $\mathrm{M}, \mathrm{V}$ and $\mathrm{d}$ represent the atomic mass, the valence and the density of substrate, respectively.

The corrosion protection efficiency (P.E.\%) was determined from the measured $I_{\text {corr }}$ using the following equation:

$$
\text { P.E. } \%=\left(1-\frac{I_{\text {corr }}^{\mathrm{c}}}{I_{\text {corr }}^{0}}\right) \times 100
$$

where $I_{\text {corr }}^{0}$ is the corrosion current of bare $\mathrm{Al}$ substrate, and $I_{\text {corr }}^{\mathrm{c}}$ is the corrosion current of the coated $\mathrm{Al}$ substrate determined by the intersection of the linear portions of the anodic and cathodic curves.

\section{Results and discussion}

\subsection{Wettability of composite coating}

The effect of PANI content on the wettability of composite coatings was 
investigated in Fig. 2a. The pure EP coating gives a WCA of $93 \pm 1^{\circ}$ due to its intrinsic hydrophilic property and smooth surface. It is found that the superhydrophobic surface with WCAs from $150^{\circ}$ to $158^{\circ}$ and SA below $5^{\circ}$ can be achieved as the PANI content increased from 0 to 9 wt $\%$ (Fig. $2 \mathrm{a}$ and $2 \mathrm{~b}$ ). With the increase of PANI content from 0 to $9 \mathrm{wt} \%$, the glycerol CAs increase continuously from 143 to $153^{\circ}$. The sliding angles of both water and glycerol droplets gradually decrease with increasing PANI content, possibly due to the increased surface roughness of the composite coating. Superamphiphobic surface with both water contact angle $\left(158 \pm 0.7^{\circ}\right)$ and glycerol contact angle $\left(151 \pm 0.6^{\circ}\right)$ larger than $150^{\circ}$ can be reached at PANI content of $4.5 \mathrm{wt} \%$ and beyond. Therefore, the composite coating with $4.5 \mathrm{wt} \%$ PANI (named as EP-4.5) was selected and investigated in detail for the following studies.

Moreover, the effect of solution $\mathrm{pH}$ (1-14) on the superhydrophobicity of EP-4.5 coating was evaluated, as shown in Fig. 2c and 2d. The high CAs (151-158 $)$ and low SAs $\left(2.1-4.3^{\circ}\right)$ reveal that the composite coating maintains its high water-repellent capability even after exposing to corrosive acidic and alkali solutions.
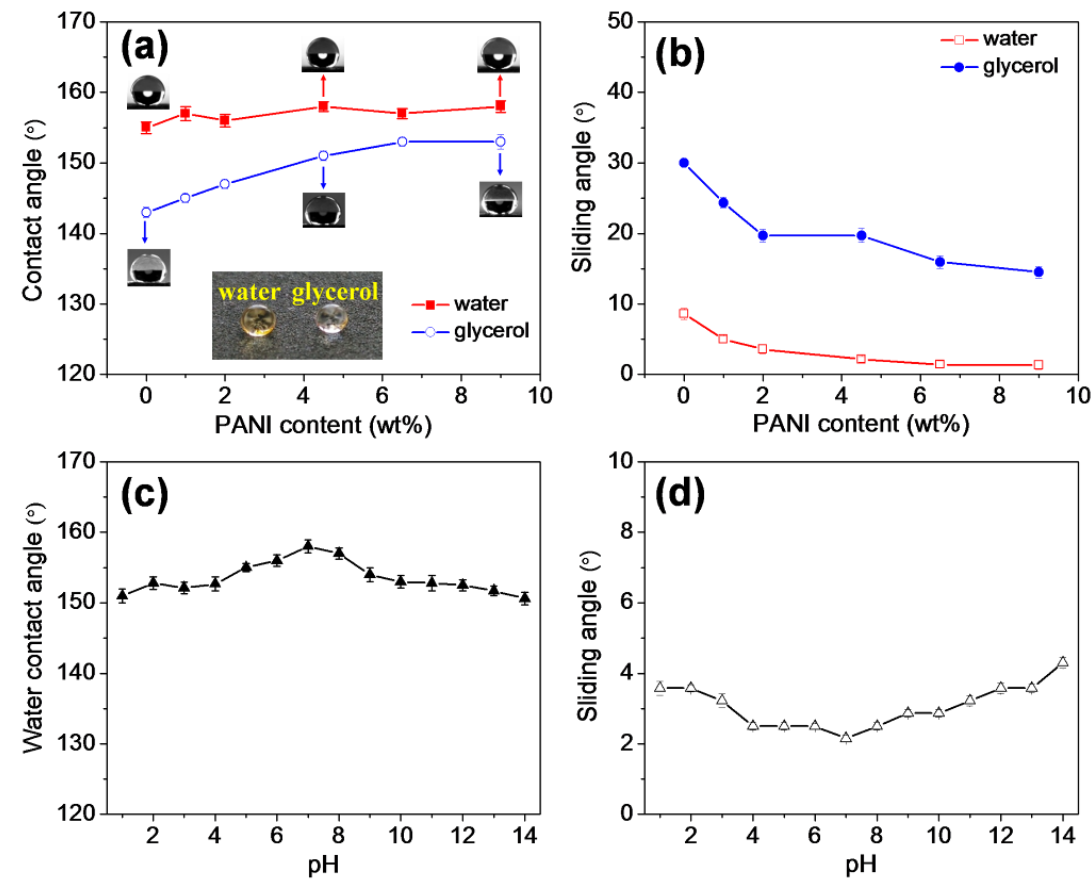

Fig. 2. Effect of PANI content on the wettability of the EP composite coatings (a, b); Effect of solution $\mathrm{pH}$ on the superhydrophobicity of the EP composite coating with $4.5 \mathrm{wt} \%$ PANI (c, d). 


\subsection{Microscopic structure of coating surface}

Fig. 3a displays the as-synthesized PANI in short fiber morphology. PANI has three reversible oxidation states: leucoemeraldine (LE), emeraldine (EM) and pernigraniline (PE). ${ }^{32}$ Only the conducting EM salt with the imine nitrogens protonated by acids can provide high anticorrosion performance via anodic protection. $^{7}$ The doping state of PANI was confirmed by the visible spectrum as shown in Fig. S3. The intense peak centered at about $900 \mathrm{~nm}$ with a free carrier tail extending into the near-infrared region indicates the conductive emeraldine salt of PANI. ${ }^{33,34}$ XPS results (Fig. S4) reveal that the as-prepared PANI is mainly composed of $\mathrm{C}, \mathrm{N}, \mathrm{O}$ and $\mathrm{Cl}$ elements. After thermal heating at $150{ }^{\circ} \mathrm{C}$ for $3 \mathrm{~h}$, the atomic percentage of $\mathrm{Cl}^{-}$decreases slightly from 3.9 to $3.5 \%$. The doping level of PANI before and after thermal treatment was analyzed by deconvolution of N1s peak, where the ratio of $\mathrm{N}^{+} / \mathrm{N}$ represents the doping level of PANI. The $\mathrm{N}^{+} / \mathrm{N}$ ratio decreases from 0.24 to 0.23 after thermal treatment, indicating the doping level of PANI can be maintained during curing process.
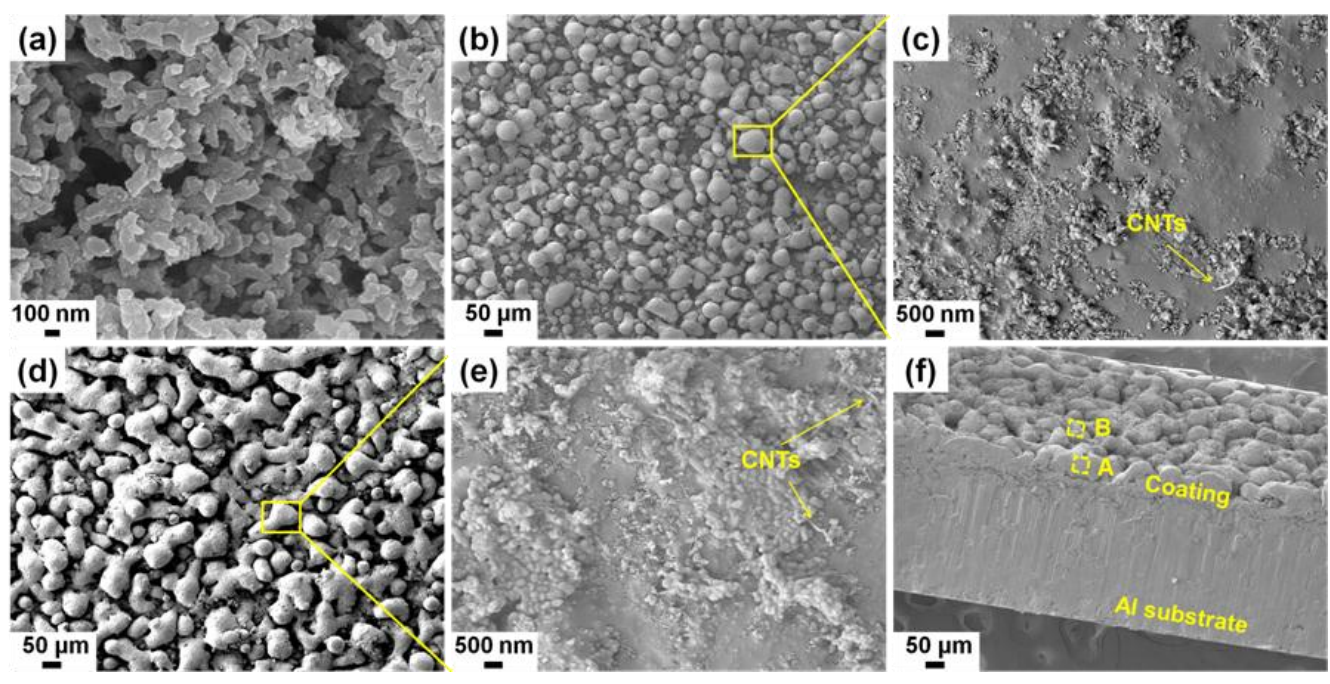

Fig. 3. The SEM surface morphologies of pure PANI (a), EP-0 (b, c) and EP-4.5 (d, e). The cross-section SEM image of EP-4.5 (f).

Manufacturing biomimetic nano/micro hierarchical surface structures is one of the major research efforts to achieve superamphiphobicity. In this work, the addition of $4.5 \mathrm{wt} \%$ PANI leads to the surface morphology transition from micro-sized 
individual spherical particles (Fig. 3b) to interconnected elliptic domains (Fig. 3d). It is worth mentioning that the EP-4.5 composite coating exhibits higher roughness $(4.7 \pm 0.1 \mu \mathrm{m})$ than the coating without PANI $(4.3 \pm 0.1 \mu \mathrm{m})$. The nano-sized particles are shown on the micro-scale protrusions (Fig. 3c and 3e). The cross-section view of EP-4.5 clearly reveals a hierarchical architecture similar to the natural lotus leaf (Fig. 3f), which well explains the superamphiphobic property of the composite film from structural perspective. Fig. $4 \mathrm{a}$ and $4 \mathrm{~b}$ shows EDS spectra of the underneath and surface section of EP-4.5 composite coating. The appearance of fluorine is attributed to the addition of FEP, and the fraction of fluorine increases from 21.31 to $39.37 \%$ from coating underneath to surface. The successful modification of low surface energy material FEP as well as the nano/micro roughness of the coating contributes to the superamphiphobic surface of EP-4.5.

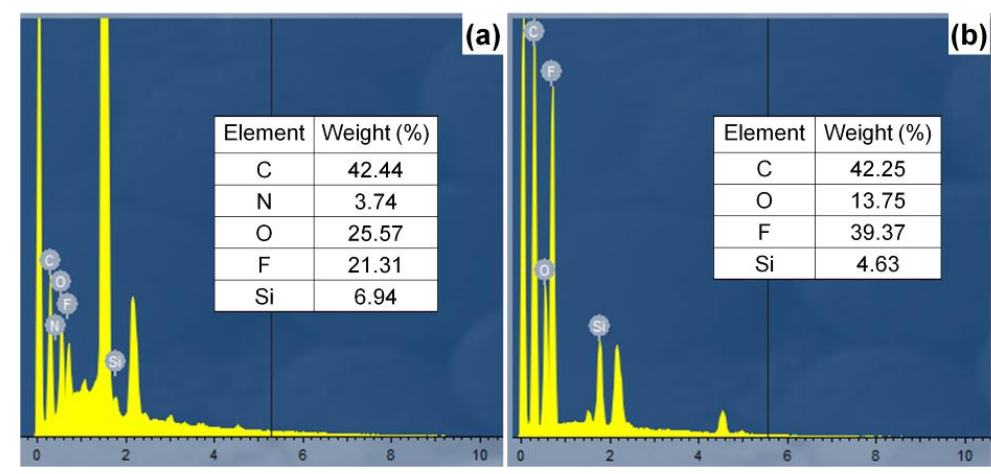

Fig. 4. EDS spectra of EP-4.5 composite coating at underneath section (a) and surface section (b) as marked area "A" and area "B" in Fig. 3f.

\subsection{Coating composition and crystalline structure analysis}

The FTIR spectrum in Fig. 5a confirms the doped form of the as-prepared PANI materials. Specifically, the absorption peak at $3430 \mathrm{~cm}^{-1}$ is assigned to $\mathrm{N}-\mathrm{H}$ stretching of aromatic amine [35]. The characteristic bands at 1575 and $1475 \mathrm{~cm}^{-1}$ are due to the $\mathrm{C}=\mathrm{C}$ ring stretching of quinoid and benzenoid structure, respectively. The adsorption bands at 1303 and $1240 \mathrm{~cm}^{-1}$ are related to the C-N stretching and $\mathrm{C}-\mathrm{H}$ stretching vibration with aromatic conjugation, respectively [36,37]. The peak observed at 1658 $\mathrm{cm}^{-1}$ denotes the $\mathrm{C}=\mathrm{N}$ stretching of the quinoid ring. The peaks at 1140 and $802 \mathrm{~cm}^{-1}$ are recognized with the $\mathrm{C}-\mathrm{H}$ in-plane bending mode and the $\mathrm{C}-\mathrm{H}$ out-of-plane bending 
mode for a 1,4 substituted aromatic ring [38].

For the EP-4.5 composite coating, the strong adsorption bands at 3439 and 2922 $\mathrm{cm}^{-1}$ are associated with the stretching vibration of $-\mathrm{OH}$ and $\mathrm{C}-\mathrm{H}$, which are generated from EP molecule. The peak at $1608 \mathrm{~cm}^{-1}$ corresponds to $\mathrm{N}-\mathrm{H}$ band of primary amines from the curing agent. The absorption bands at around 1462 and $830 \mathrm{~cm}^{-1}$ are related to the characteristic absorption peaks of benzene ring and epoxide ring in epoxy resin, respectively [39]. The adsorption peak at $1038 \mathrm{~cm}^{-1}$ in the spectrum of pure EP is due to the aliphatic carbon-oxygen stretching $\left(-\mathrm{O}-\mathrm{CH}_{2}-\right)$. In the spectrum of EP-4.5 composite coating, the significant decrease of this peak indicates the curing of epoxy. The bands at 1240 and $1156 \mathrm{~cm}^{-1}$ can be attributed to the stretching vibration of the $-\mathrm{CF}_{3}$ and $-\mathrm{CF}_{2}$ in FEP molecules [27]. Moreover, the peak at $640 \mathrm{~cm}^{-1}$ indicates the $-\mathrm{CF}_{2}$ rocking or wagging-bending vibration in FEP molecule [40].

The XRD patterns of pure PANI, FEP, EP powder and EP-4.5 composite coating are shown in Fig. $4 \mathrm{~b}$. The diffraction peak at $2 \theta=21^{\circ}$ and $25^{\circ}$ can be attributed to the characteristic peak of $\mathrm{SiO}_{2}$ and PANI, respectively. The sharp peak at $2 \theta=17.8^{\circ}$ can be ascribed to the existence of FEP. Other significant diffraction peaks at $2 \theta=27^{\circ}, 35.8^{\circ}$ and $54^{\circ}$ are associated with the crystallization of epoxy resin during thermal curing.
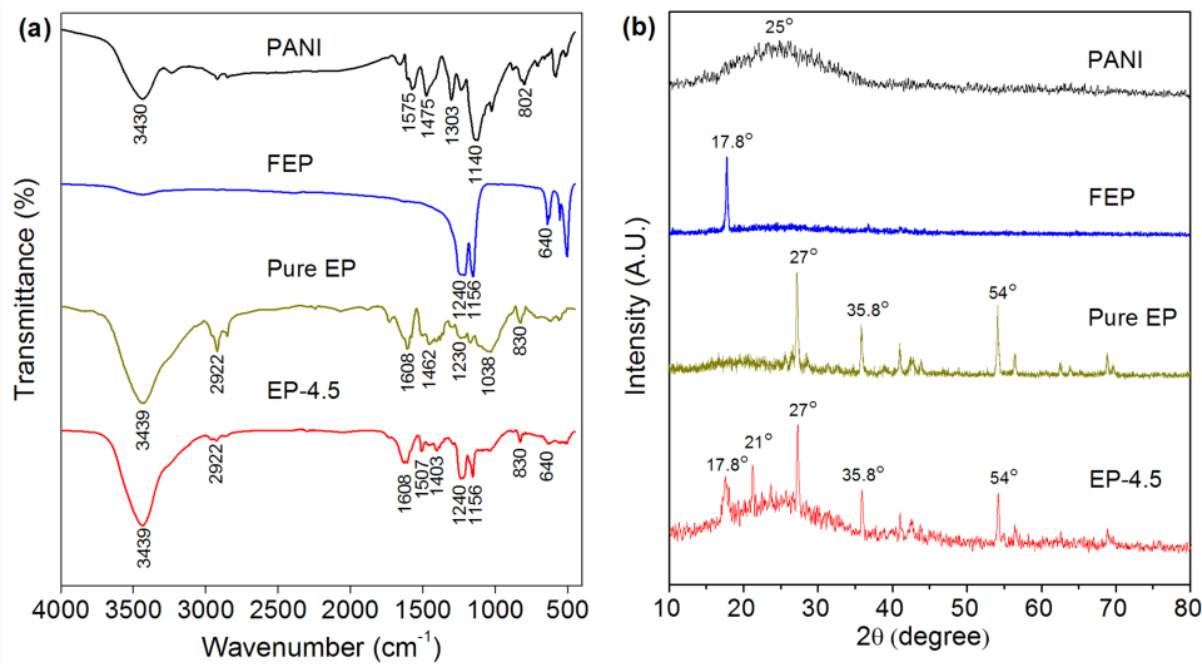

Fig. 5. FT-IR (a) and XRD (b) spectra of the as-prepared PANI materials, FEP, pure EP powder and EP-4.5 composite coating.

\subsection{Coating adhesion evaluation}


Adhesion is an important index to determine the physical barrier function of coatings. Strong adhesion restricts the fast diffusion of electrolyte and prevents spreading of corrosion from the spot corrosion. The EP-4.5 composite coating was scribbled into $2 \times 2 \mathrm{~mm}$ gridding using a razor to expose the base metal, and then adhesive tape was pressed and pulled to remove coating from the scored surface within 5 min. According to standard GB/T 9286, Grade 0 indicates the best adhesion property of the coating, while Grade 5 stands for the worst adhesion result. As shown in Fig. 6a, the EP-4.5 composite coating has little peeling at the intersection of the scratches with impacted area in the cross incision less than 5\%. This coating can be classified as Grade 1, which implies that it meets the requirements in practical application. The strong interface adhesive strength of the composite coating is mainly attributed to the highly cross-linked interfacial layer with reactions between hydroxyls on the Al surface and epoxy groups of EP molecule [29].
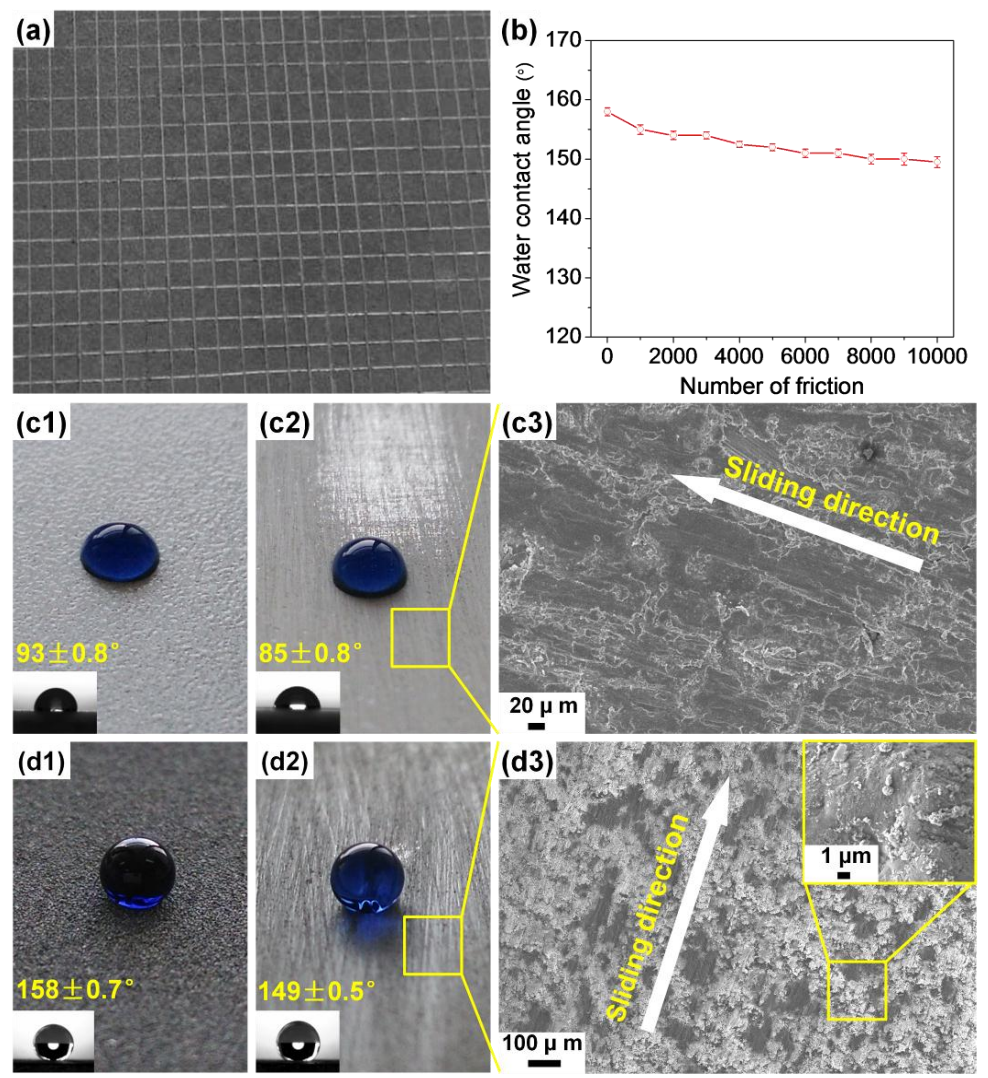

Fig. 6. The adhesive ability of the EP-4.5 composite coating (a), influence of the abrasion on the wettability of the EP-4.5 coating (b), the optical and SEM images before and after rubbing the pure EP coating (c1, c2, c3) and the EP-4.5 composite coating (d1, d2, d3). 


\subsection{Wear-resistance of composite coating}

In practical application, a robust surface is essential to maintain surface function throughout the service life of coating [41,42]. In order to evaluate the wear-resistance of the superamphiphobic coating, abrasion test on EP-4.5 was performed and compared with pure EP coating. The influence of abrasion on wettability of the coatings is given in Fig. 6b. Apparently, the WCA of pure EP coating reduces from $93 \pm 0.5^{\circ}$ to $85 \pm 0.5^{\circ}$ due to the reduced surface roughness after abrasion test (Fig. $6 \mathrm{c}$ ). While, the WCA of EP-4.5 surface decreases slightly from $158 \pm 0.7^{\circ}$ to $149 \pm 0.5^{\circ}$ after 10000 times abrasion (Fig. 6d). Based on the SEM observation, new micro-scaled roughness profiles were created by abrasion and the embedded nanoparticles were exposed at the air-interface, resulting in a new nano/micrometer level roughness. It is suggested that the large amount of air entrapped in the rough surface is responsible for the high water-repellency after abrasion.

In 2010, Xiu et al. found the importance of dual-scale hierarchical structures for superhydrophobic surfaces [43]. In 2014, Esteves et al. found that the recreation of multi-scale surface topographies upon repetitive damage of the superhydrophobic surface is actually beneficial to maintain the surface anti-wettability [41]. In this work, the excellent wear-resistance of the superamphiphobic surface is not only contributed by the regenerated nano/micro-structures but also the self-lubrication effect of embedded FEP and CNTs [44,45].
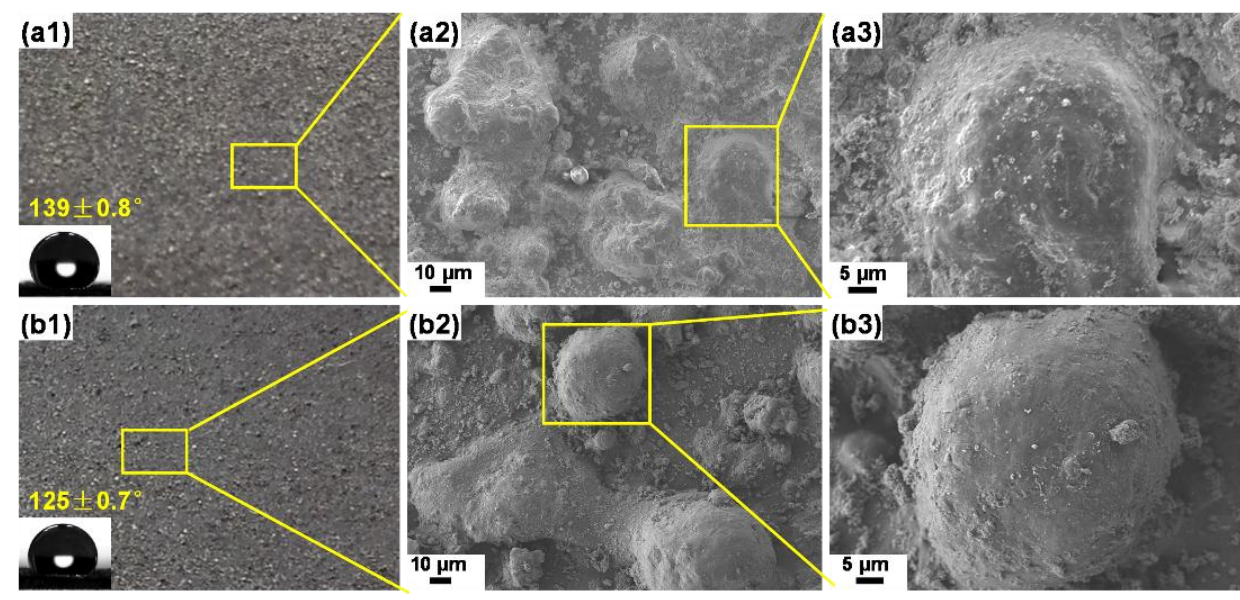

Fig. 7. SEM images of the coatings immersed in the strongly acidic $(\mathrm{pH}=1)$ (a) and alkaline ( $\mathrm{pH}=14)(\mathrm{b})$ solutions for 90 days. 


\subsection{Anticorrosion performance of the coating}

\subsubsection{Corrosion resistance against corrosive solutions}

After immersing EP-4.5 in the strongly acidic $(\mathrm{pH}=1)$ or alkaline $(\mathrm{pH}=14)$ solution for 90 days, the WCAs on the coating retained $139 \pm 0.8^{\circ}$ and $125 \pm 0.7^{\circ}$, respectively. The SEM images of the EP-4.5 composite coating are displayed in Fig. 7. It can be observed that the roughness of both coatings can be maintained although the nanoparticles on the micro papillae diminished to some extent. These results reveal the superior pH-resistant property of the composite coating and suggest its applicability in harsh corrosive environment.

\subsubsection{Potentiodynamic polarization analysis}

We investigated the corrosion resistance of the superamphiphobic coating in 3.5 wt\% $\mathrm{NaCl}$ aqueous solution. The typical potentiodynamic polarization curves obtained on uncoated and coated Al substrates are displayed in Fig. 8, and the electrochemical parameters obtained from the polarization curves are summarized in Table 1. As compared to the bare Al substrate, both EP-0 and EP-4.5 coated samples exhibit significant shift of corrosion potential ( $\left.E_{\text {corr }}\right)$ to the positive direction, indicating the improved corrosion resistance. These results are in good agreement with our previous observations in organic superhydrophobic coating systems [27]. Moreover, the $E_{\text {corr }}$ value of EP-4.5 after immersing in $\mathrm{NaCl}$ solution for 1 day and 30 days are estimated to be about -215 and $-457 \mathrm{mV}$ respectively, which is much lower than the EP-0 coated substrate of -332 and $-726 \mathrm{mV}$. The higher $E_{\text {corr }}$ could be attributed to the higher redox potential of PANI filler in the coating.

The large current density of the uncoated $\mathrm{Al}$ substrate $\left(1.4 \times 10^{-5} \mathrm{~A} / \mathrm{cm}^{2}\right)$ is attributed to the pitting corrosion as confirmed by the following EIS results. It implies that the corrosive chloride ions has permeated through the thin passivation film and consequently initiated pitting corrosion. Both anodic and cathodic current densities of EP-0 and EP-4.5 decrease by almost 4 orders of magnitude after $1 \mathrm{~d}$ immersion in $\mathrm{NaCl}$ solution. In particular, the corrosion current of EP-4.5 coated substrate is as low 
as $7.5 \times 10^{-9} \mathrm{~A} / \mathrm{cm}^{2}$ after $30 \mathrm{~d}$ immersion, implying the extremely low corrosion rate (0.088 $\mu \mathrm{m} /$ year) and high protection efficiency $(99.946 \%)$. All these results demonstrate that the robust superamphiphobic coating containing conductive PANI can provide an enhanced and durable corrosion protection for the Al substrate.

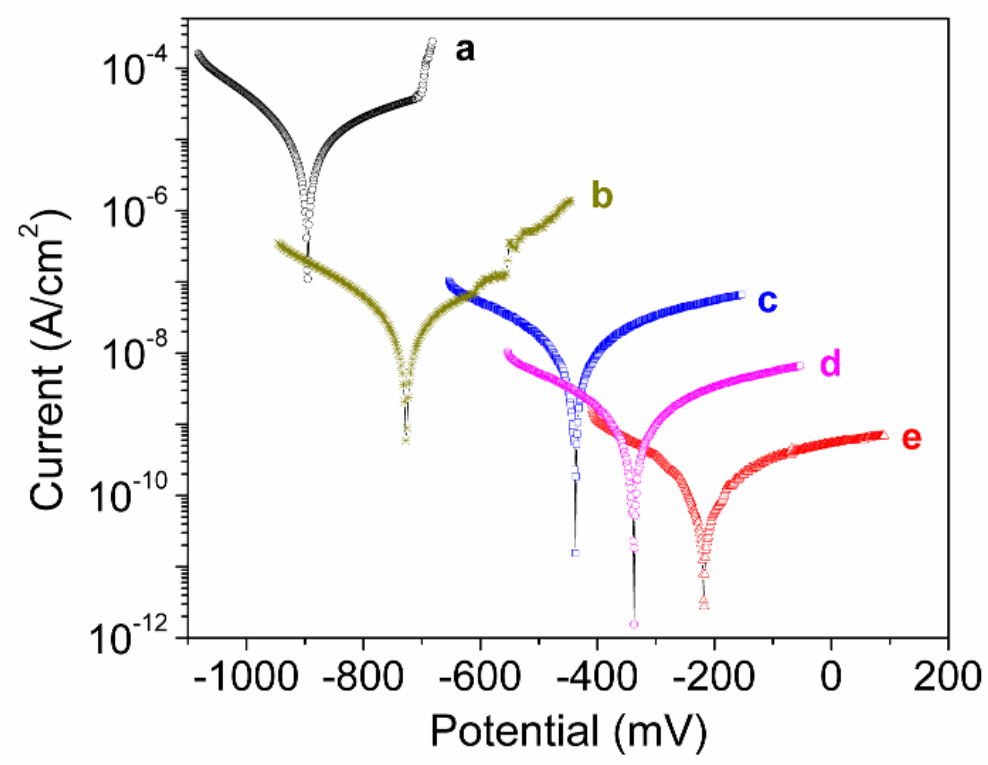

Fig. 8 Potentiodynamic polarization curves for the untreated aluminum plate after immersion in $3.5 \mathrm{wt} \% \mathrm{NaCl}$ aqueous solution for $0.5 \mathrm{~h}$ (a), the superamphiphobic composite coating without PANI (EP-0) after $1 \mathrm{~d}$ (d) and $30 \mathrm{~d}$ (b) immersion in $3.5 \mathrm{wt} \% \mathrm{NaCl}$ solution and the superamphiphobic composite coating with $4.5 \mathrm{wt} \%$ PANI (EP-4.5) after $1 \mathrm{~d}$ (e) and $30 \mathrm{~d}$ (c) immersion in $3.5 \mathrm{wt} \% \mathrm{NaCl}$ solution.

\section{Table 1}

Potentiodynamic polarization parameter values for uncoated and coated Al substrates.

\begin{tabular}{cccccc}
\hline Sample & Immersion time & $E_{\text {corr }}(\mathrm{mV})$ & $I_{\text {corr }}\left(\mathrm{A} / \mathrm{cm}^{2}\right)$ & $\mathrm{CR}(\mu \mathrm{m} /$ year $)$ & P.E. $(\%)$ \\
\hline Uncoated & $0.5 \mathrm{~h}$ & -895 & $1.4 \times 10^{-5}$ & 163.94 & - \\
& $1 \mathrm{~d}$ & -332 & $1.8 \times 10^{-9}$ & 0.021 & 99.987 \\
EP-0 & $30 \mathrm{~d}$ & -726 & $1.1 \times 10^{-7}$ & 1.288 & 99.214 \\
& $1 \mathrm{~d}$ & -215 & $3.1 \times 10^{-10}$ & 0.004 & 99.998 \\
EP-4.5 & $30 \mathrm{~d}$ & -457 & $7.5 \times 10^{-9}$ & 0.088 & 99.946 \\
\hline
\end{tabular}




\subsubsection{EIS analysis}

To unveil the anticorrosion mechanism and quantify the corrosion resistant performance of the superamphiphobic coating, EIS studies were performed in this work. For uncoated aluminum substrate, the Nyquist curve shows a capacitive loop at high and medium frequency range (Fig. 9a). The semi-circles rolls over and extends to the fourth quadrant, and a pseudo-inductive loop at low frequency end can be observed, indicating that the faradic process is taking place on the free electrode sites. This inductive loop is generally attributed to the adsorption of species resulting from the $\mathrm{Al}$ dissolution and the adsorption of chloride ions [46]. One well-defined time constant at around $10 \mathrm{~Hz}$ is shown in the Bode plot of the uncoated Al substrate, indicating the corrosion process occurred at the metal surface.
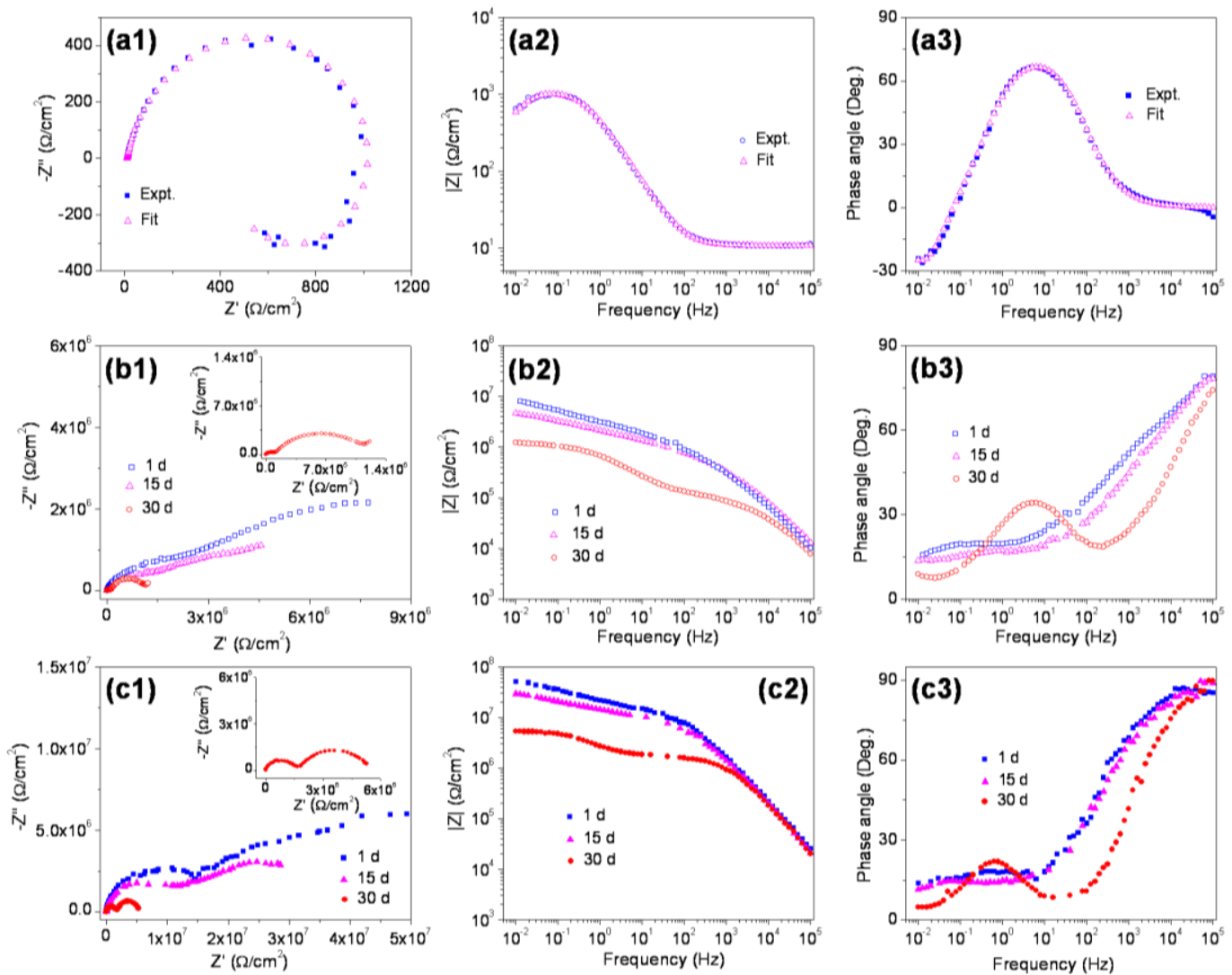

Fig. 9. Nyquist plots and Bode plots for the bare Al substrate (a), EP-0 (b) and EP-4.5 (c) coatings as a function of exposure time.

The impedance spectra of both EP-0 and EP-4.5 have capacitive loops at high 
frequencies and show an approximately linear relation between the real and imaginary parts of the impedance at medium and low frequencies within $15 \mathrm{~d}$ immersion, while two capacitive loops can be observed after $30 \mathrm{~d}$ immersion (Fig. 9b and 9c). The capacitive loop at medium frequencies is attributed to the charge transfer of the corrosion across the corrosion layer and the double layer capacitance at the electrode surface. The loop at low frequencies is attributed to the charge transfer resistance [17]. The decrease in capacitive loops indicates the gradual reduction of anticorrosion performance of the coating, which is consistent with the results obtained from potentiodynamic polarization curves. The reduced corrosion protection is attributed to the penetration of corrosive chloride ions to the coating/aluminum interface and initiation of localized corrosion [47].

The impedance decreases gradually with extending immersion time. The highest impedance level of the superamphiphobic EP-4.5 coating indicates a high corrosion resistance due to its excellent water-repellency property. The $|\mathrm{Z}|$ value of EP-4.5 at low frequency after $30 \mathrm{~d}$ immersion is estimated to be about 3 orders of magnitude higher than that of the untreated $\mathrm{Al}$ substrate. This indicates that the superamphiphobic surface considerably retards the corrosion process.

The superamphiphobic surfaces after $15 \mathrm{~d}$ immersion in $3.5 \mathrm{wt} \% \mathrm{NaCl}$ solution exhibit one phase maxima at high frequencies around $10^{5} \mathrm{~Hz}$, which is associated to the responses of the electrolyte/coating interface. While the coating after $30 \mathrm{~d}$ immersion exhibits two phase maxima at low and high frequencies, respetively. The time constant appearing at low frequency can be ascribed to the capacitance of double layer, which is an indicator of the on-going corrosion process at the electrolyte/substrate interface. In the case of EP-4.5, the time constant shifted to lower frequency than that of EP-0 and untreated Al substrate, revealing good barrier properties of the superamphiphobic coating that suppresses the penetration of the $\mathrm{NaCl}$ aqueous solution to the $\mathrm{Al}$ surface.

To precisely quantify the different corrosion behavior of uncoated and superamphiphobically coated aluminum substrates, two different equivalent circuit models are used in this work. The equivalent circuit model in Fig. 10a is applied to 
simulate the electrochemical behavior of the bare Al substrate. The model consists of the solution resistance $\left(R_{\mathrm{S}}\right)$, the charge transfer resistance of the interfacial corrosion reaction $\left(R_{\mathrm{ct}}\right)$, the inductance $(L)$, the inductive resistance $\left(R_{\mathrm{L}}\right)$ and the constant phase element $(C P E)$ of the coating $\left(Q_{\mathrm{c}}\right)$. The $C P E$ is used instead of pure capacitor to fit the impedance behavior of the electrical double layer more accurately. Well fitted results are obtained as shown in Fig. 9a, and the fitted parameters are summarized in Table 2. The large $Q_{\mathrm{dl}}$ value of $5.5 \times 10^{-5} \mathrm{~F} / \mathrm{cm}^{2}$ may be attributed to the increased local dielectric constant and/or the decreased electrical double layer thickness, indicating the adsorption of corrosive chloride ions at the metal/solution interface [46].

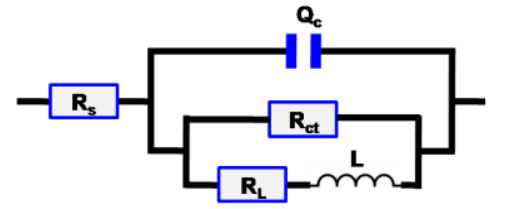

(a)

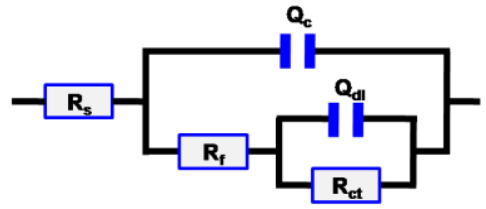

(b)

Fig. 10. Equivalent circuit models of the studied system for uncoated (a) and (b) coated aluminum substrates after immersion in $3.5 \mathrm{wt} \% \mathrm{NaCl}$ aqueous solution.

Since the rough superamphiphobic coating possesses many micropores that could trap air at the solid-liquid interface, a different equivalent circuit model including the impedance of the superhydrophobic film $R_{\mathrm{f}}$ is used as shown in Fig. 10b [16]. This model is commonly used to simulate the corrosion behavior of the coated metal substrates [8,48]. $R_{\mathrm{s}}, R_{\mathrm{ct}}, Q_{\mathrm{c}}$ and $Q_{\mathrm{dl}}$ represent the solution resistance, charge transfer resistance, the $\mathrm{CPE}$ of the coating and the double layer capacitance, respectively. The $R_{\mathrm{ct}} \| Q_{\mathrm{dl}}$ elements in Fig. 10b mean the impedance with the interface reaction between the coating and aluminum plate. As an indicator of total corrosion resistance performance, the change of $R_{\mathrm{ct}}$ represents the diffusion of the corrosion reactants and the reaction kinetics at the surface of the electrode [46]. Higher $R_{\mathrm{ct}}$ value is obtained for the substrate coated with EP-4.5, indicating its better corrosion protection performance. The $R_{\mathrm{f}}$ value is related to the number of pores or capillary channels perpendicular to the substrate surface, through which the electrolyte reaches the interface [31]. The $R_{\mathrm{f}}$ value of EP-4.5 is much higher than that of EP-0, indicating its 
much less porous structures. The lower $R_{\mathrm{f}}$ values after $30 \mathrm{~d}$ immersion reveal that some pores are opened and the electrolyte species may permeate the coating.

Generally, the increase in $Q_{\mathrm{dl}}$ value of EP-0 and EP-4.5 is related to the diffusion of electrolyte at the interface and resultant increase of delaminated area [31]. The coating with PANI exhibits the lowest $Q_{\mathrm{dl}}$ value that reveals a relatively stable coating/metal interface.

According to the above electrochemical tests, the corrosion protection performance of the tested sampels in $3.5 \mathrm{wt} \% \mathrm{NaCl}$ solution can be established in the following order: EP-4.5 > EP-0 $\gg$ uncoated $\mathrm{Al}$ substrate. The enhanced anticorrosive performance of EP-4.5 composite coating could be attributed to the excellent physical barrier effect of superamphiphobic surface and the catalytic redox effect of electroactive PANI, which facilitates the formation of protective passivation aluminum hydroxide layer [11].

\section{Table 2}

Parameter values of uncoated $\mathrm{Al}$ substrate.

\begin{tabular}{ccccccc}
\hline Parameter & $R_{\mathrm{s}}\left(\Omega / \mathrm{cm}^{2}\right)$ & $Q_{\mathrm{dl}}\left(\mathrm{F} / \mathrm{cm}^{2}\right)$ & $\mathrm{n}_{\mathrm{dl}}$ & $R_{\mathrm{ct}}\left(\Omega / \mathrm{cm}^{2}\right)$ & $R_{\mathrm{L}}\left(\Omega / \mathrm{cm}^{2}\right)$ & $L(\mathrm{H})$ \\
\hline Value & 74.95 & $5.5 \times 10^{-5}$ & 0.86 & $2.7 \times 10^{3}$ & $4.7 \times 10^{3}$ & $3.7 \times 10^{4}$ \\
\hline
\end{tabular}

\section{Table 3}

Parameter values of coated $\mathrm{Al}$ substrates.

\begin{tabular}{cccccc}
\hline Sample & Immersion time & $R_{\mathrm{f}}\left(\Omega / \mathrm{cm}^{2}\right)$ & $R_{\mathrm{ct}}\left(\Omega / \mathrm{cm}^{2}\right)$ & $Q_{\mathrm{c}}\left(\mathrm{F} / \mathrm{cm}^{2}\right)$ & $Q_{\mathrm{dl}}\left(\mathrm{F} / \mathrm{cm}^{2}\right)$ \\
\hline \multirow{2}{*}{ EP-0 } & $1 \mathrm{~d}$ & $1.1 \times 10^{6}$ & $1.3 \times 10^{7}$ & $3.9 \times 10^{-9}$ & $1.9 \times 10^{-7}$ \\
& $15 \mathrm{~d}$ & $9.8 \times 10^{5}$ & $5.2 \times 10^{6}$ & $4.1 \times 10^{-9}$ & $2.9 \times 10^{-7}$ \\
& $30 \mathrm{~d}$ & $1.5 \times 10^{5}$ & $1.0 \times 10^{6}$ & $1.5 \times 10^{-8}$ & $3.0 \times 10^{-7}$ \\
& $1 \mathrm{~d}$ & $6.4 \times 10^{6}$ & $1.0 \times 10^{8}$ & $1.7 \times 10^{-10}$ & $3.0 \times 10^{-8}$ \\
$\mathrm{EP}-4.5$ & $15 \mathrm{~d}$ & $3.7 \times 10^{6}$ & $5.7 \times 10^{7}$ & $1.9 \times 10^{-10}$ & $4.6 \times 10^{-8}$ \\
& $30 \mathrm{~d}$ & $1.6 \times 10^{6}$ & $4.1 \times 10^{6}$ & $2.2 \times 10^{-10}$ & $1.6 \times 10^{-7}$ \\
\hline
\end{tabular}

\section{Conclusions}

In conclusion, we have successfully fabricated a novel superamphiphobic and 
electroactive EP/PANI/FEP/CNTs/SiO ${ }_{2}$ composite coating with designed nano/micro-structured surface by facile and scalable air spraying and electrostatic spraying processes. The major conclusions are summarized as follow:

(1) The increasing addition of PANI in $\mathrm{EP} / \mathrm{FEP} / \mathrm{CNTs} / \mathrm{SiO}_{2}$ improves the anti-wettability of the composite coating. By adding 4.5 wt $\%$ PANI, superamphiphobic property can be achieved. The superhydrophobic property with WCAs greater than $150^{\circ}$ can be maintained even in the presence of corrosive media with $\mathrm{pH}$ from 1 to 14 .

(2) The as-prepared coating with conductive PANI possesses excellent wear-resistance, with high WCA $\left(149 \pm 0.5^{\circ}\right)$ after 10000 times abrasion due to the regeneration of nano/micro-structures. The coating exhibits high water repellency after immersion in acidic $(\mathrm{pH}=1)$ and alkaline solutions $(\mathrm{pH}=14)$, indicating its durability in corrosive environment. The strong adhesion of the prepared coating on Al substrate (Grade 1 according to GB/T 9286) could benefit the strength and durability of the coatings in practical application.

(3) Electrochemical corrosion results reveal that the superamphiphobic coating containing $4.5 \mathrm{wt} \%$ PANI exhibits much higher corrosive resistance property than that of the coating without PANI and the uncoated $\mathrm{Al}$ plate. The enhanced anticorrosion behavior could be attributed to the better barrier effect of superamphiphobic surface and the anodic protection as well as the catalytically passivation of aluminum-coating interface.

It is believed that this electroactive/superamphiphobic multifunctional composite coating processed by simple spraying will generate great impact in corrosion protection of various metallic surfaces.

\section{Acknowledgements}

The authors acknowledge the support from the start-up fund of The University of Akron, National Science Foundation of China (51175066, 21507009), China Postdoctoral Science Foundation (2014M551215), Heilongjiang Educational Committee Foundation (12531077), PetroChina Innovation Foundation and Science 
Foundation of Northeast Petroleum University (2013NQ111). Acknowledgement is also made to the Donors of the American Chemical Society Petroleum Research Fund for support of this research (\#55570-DNI10). Authors are also thankful to Dr. Zhorro (George) Nikolov in National Polymer Innovation Center (Univeristy of Akron) for carrying out XPS measurements of the samples.

\section{References}

[1] F. Khelifa, S. Ershov, M.-E. Druart, Y. Habibi, D. Chicot, M.-G. Olivier, R. Snyders, P. Dubois. J. Mater. Chem. A 3 (2015) 15977-15985.

[2] H. Wei, Y. Wang, J. Guo, N.Z. Shen, D. Jiang, X. Zhang, X. Yan, J. Zhu, Q. Wang, L. Shao, H. Lin, S. Wei, Z. Guo. J. Mater. Chem. A 3 (2015) 469-480.

[3] O. Jilani, N. Njah, P. Ponthiaux. Corros. Sci. 87 (2015) 259-264.

[4] Z. Tian, H. Yu, L. Wang, M. Saleem, F. Ren, P. Ren, Y. Chen, R. Sun, Y. Sun, L. Huang. RSC Adv. 4 (2014) 28195-28208.

[5] A. Ghazi, E. Ghasemi, M. Mahdavian, B. Ramezanzadeh, M. Rostami. Corros. Sci. 94 (2015) $207-217$.

[6] F. Khelifa, M.-E. Druart, Y. Habibi, R. Rioboo, M. Olivier, J.D. Coninck, P. Dubois. J. Mater. Chem. A 1 (2013) 10334-10344.

[7] H. Lu, Y. Zhou, S. Vongehr, K. Hu, X. Meng. Synthetic Met. 161 (2011) 1368-1376.

[8] X. Li, M. Rao, H. Lin, D. Chen, Y. Liu, S. Liu, Y. Liao, L. Xing, M. Xu, W. Li. J. Mater. Chem. A 3 (2015) 18098-18104.

[9] C. Dispenza, M.A. Sabatino, N. Deghiedy, M.P. Casalettom, G. Spadaro, S. Piazza, H.A.A. El-Rdhim. Polymer 67 (2015) 128-138.

[10] J. Zhu, M. Chen, H. Qu, X. Zhang, H. Wei, Z. Luo, H.A. Colorado, S. Wei, Z. Guo. Polymer 53 (2012) 5953-5964.

[11] C.J. Weng, J.Y. Huang, K.Y. Huang, Y.S. Jhuo, M.H. Tsai, J.M. Yeh. Electrochim. Acta 55 (2010) $8430-8438$.

[12] T.C. Huang, T.C. Yeh, H.Y. Huang, W.F. Ji, Y.C. Chou, W.I. Hung, J.M. Yeh, M.H. Tsai. Electrochim. Acta 56 (2011) 10151-10158. 
[13] K.Y. Huang, C.L. Shiu, P.S. Wu, Y. Wei, J.M. Yeh, W.T. Li. Electrochim. Acta 54 (2009) $5400-5407$

[14] A.A. Hermas, M.A. Salam, S.S. Al-Juaid, A.H. Qusti, M.Y. Abdelaal. Prog. Org. Coat. 77 (2014) 403-411.

[15] X. Bai, T.h. Tran, D. Yu, A. Vimalanandan, X. Hu, M. Rohwerder, Corros. Sci. 95 (2015) $110-116$.

[16] A.M. Kumar, Z.M. Gasem. Prog. Org. Coat. 78 (2015) 387-394.

[17] T. Ishizaki, Y. Masuda, M. Sakamoto. Langmuir 27 (2011) 4780-4788.

[18] Y. Liu, S. Li, J. Zhang, J. Liu, Z. Han, L. Ren. Corros. Sci. 94 (2015) 190 - 196.

[19] C.K. Söz, E. Yilgör, I. Yilgör. Polymer 62 (2015) 118-128.

[20] R. Yuan, H. Wang, L. Mu, L. Chen, Y. Zhu, J. Zhu. J. Mater. Chem. A 3 (2015) 1929919303.

[21] A.C.C. de Leon, R.B. Pernites, R.C. Advincula. ACS Appl. Mater. Interfaces 4 (2012) 31693176.

[22] C.W. Peng, K.C. Chang, C.J. Weng, M.C. Lai, C.H. Hsu, S.C. Hsu, Y.Y. Hsu, W.I. Hung, Y. Wei, J.M. Yeh. Electrochim. Acta 95 (2013) 192-199.

[23] T. Verho, C. Bower, P. Andrew, S. Franssila, O. Ikkala, R.H.A. Ras. Adv. Mater. 23 (2011) $673-678$.

[24] F. Xue, D. Jia, Y. Li, X. Jing. J. Mater. Chem. A 3 (2015) 13856-13863.

[25] Y. Lu, S. Sathasivam, J. Song, C.R. Crick, C.J. Carmalt, I.P. Parkin. Science 347 (2015) $1132-1135$

[26] X. Deng, L. Mammen, H.J. Butt, D. Vollmer. Science 335 (2011) 67-70.

[27] H. Wang, D. Gao, Y. Meng, H. Wang, E. Wang, Y. Zhu. Prog. Org. Coat. 82 (2015) 74-80.

[28] Z. Chu, S. Seeger. Chem. Soc. Rev. 43 (2014) 2784-2798.

[29] H. Wang, Z. Liu, E. Wang, R. Yuan, D. Gao, X. Zhang, Y. Zhu. Appl. Surf. Sci. 332 (2015) $518-524$.

[30] F. Chen, J. Song, Y. Lu, S. Huang, X. Liu, J. Sun, C.J. Carmalt, I.P. Parkin, W. Xu. J. Mater. Chem. A 3 (2015) 20999-21008.

[31] C.W. Peng, C.H. Hsu, K.H. Lin, P.L. Li, M.F. Hsieh, Y. Wei, J.M. Yeh, Y.H. Yu. Electrochim. Acta 58 (2011) 614-620. 
[32] U. Riaz, C. Nwaoha, S.M. Ashraf. Prog. Org. Coat. 77 (2014) 743-756.

[33] Y. Zhu, D. Hu, M. Wan, L. Jiang, Y. Wei. Adv. Mater. 19 (2007) 2092-2096.

[34] S. Shi, Q. Wu, R. Li, Y. Zhu, Y. Qin, C. Qiao. Nanotechnology 24 (2013) 175602.

[35] X. Zhou, Z. Zhang, X. Xu, X. Men, X. Zhu. Appl. Surf. Sci. 276 (2013) 571-577.

[36] J. Fei, Y. Cui, X. Yan, Y. Yang, K. Wang, J. Li. ACS Nano 3 (2009) 3714-3718.

[37] H. Wei, J. Zhu, S. Wu, S. Wei, Z. Guo. Polymer 54 (2013) 1820-1831.

[38] Q. Yu, J. Xu, J. Liu, B. Li, Y. Liu, Y. Han. Appl. Surf. Sci. 263 (2012) 532-535.

[39] S.S. Golru, M.M. Attar, B. Ramezanzadeh. Prog. Org. Coat. 77 (2014) 1391-1399.

[40] Q. Huang, C. Xiao, X. Hu, S. An. J. Mater. Chem. 21 (2011) 16510-16516.

[41] A.C.C. Esteves, Y. Luo, M.W.P. van de Put, C.C.M. Carcouët, G. de With. Adv. Funct. Mater. 24 (2014) 986-992.

[42] F. Wan, D.-Q. Yang, E. Sacher. J. Mater. Chem. A 3 (2015) 16953-16960.

[43] Y. Xiu, Y. Liu, D.W. Hess, C.P. Wong. Nanotechnology 21 (2010) 155705.

[44] H. Zhao, J. Zhang, T. Ji, M. Yang, M. Chao, K. Kou. Tribol. Lett. 45 (2012) 333-339.

[45] Y. Shi, X. Feng, H. Wang, X. Lu. Wear 264 (2008) 934-939.

[46] K. Shalabi, Y.M. Abdallah, A.S. Fouda. Res. Chem. Intermediat. 41 (2014) 4687-4711.

[47] M. Sababi, J. Pan, P.E. Augustsson, P.E. Sundell, P.M. Claesson. Corros. Sci. 84 (2014) $189-197$.

[48] X.M. Li, B. Faber, B. Minch, H. Castaneda. Corrosion 70 (2014) 615-626. 


\section{Graphical Abstract}

\section{Superamphiphobicity and Electroactivity Enabled Dual Physical/Chemical}

Protections in Novel Anticorrosive Nanocomposite Coatings
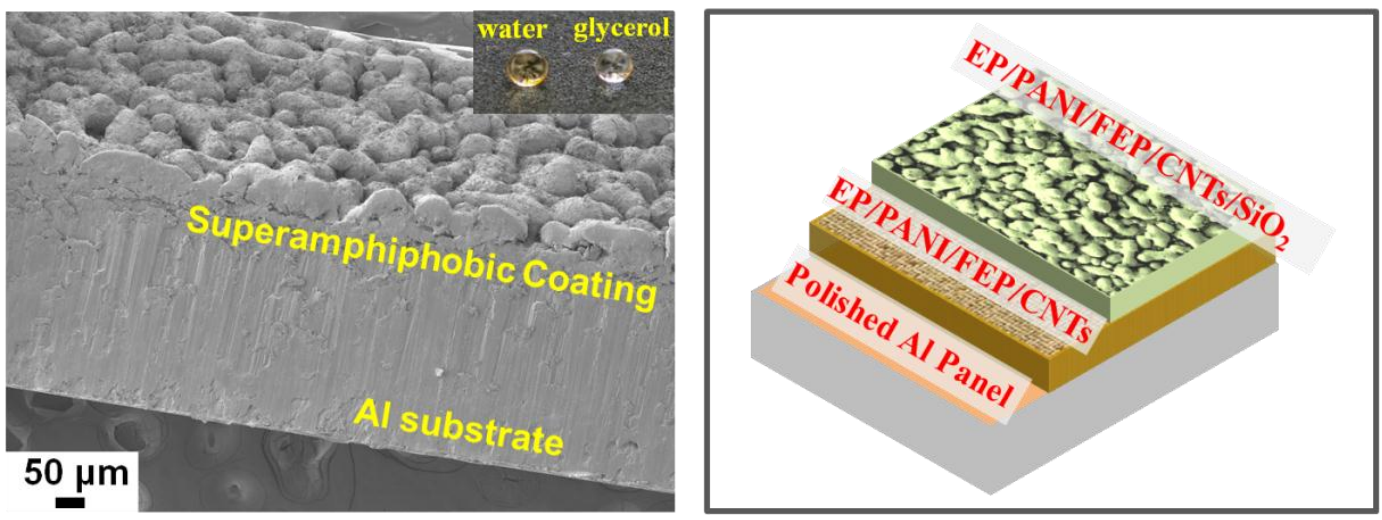

Superamphiphobic and electroactive nanocomposite coating manufactured by facile spraying processes shows excellent anticorrosion property in corrosive media due to its dual physical and chemical protections. 